\title{
Parenterale Ernährung onkologischer Patienten im Wandel
}

\author{
Wissenschaftliche Grundlagen und klinische Anwendung ${ }^{1}$
}

U. Jehn

\author{
Changing perspectives of parenteral nutrition in cancer patients. \\ Rational and clinical implications
}

Anorexie und Kachexie bei Tumorpatienten als häufigstes paraneoplastisches Syndrom $(1,2)$, gewinnen bei Onkologen wieder zunehmend an Interesse $(3,4)$. Die Pathogenese ist nach wie vor unklar.

Gewichtsverlust vor Beginn einer Chemotherapie korreliert mit dem Performance-Status (Leistungs-Index) des Tumorpatienten und ist mit einem herabgesetzten medianen Überleben verbunden (5). Komponenten der Tumorkachexie sind neben dem Gewichtsverlust, Appetitlosigkeit, Muskelatrophie, Imbalancen und Störungen im Eiweiß-, Fett und Kohlenhydrat-Metabolismus sowie eine Anämie $(6,7)$. Krebskachexie ist weit komplexer als ein chronischer Hungerzustand. Ein wesentlicher Unterschied besteht darin, dass im Hungerzustand vorzugsweise Fett verbrannt wird und Skelettmuskulatur erhalten bleibt, während bei Tumorpatienten Fett und Muskulatur gleichmäßig abgebaut werden. Studien haben gezeigt (8-13), dass ein veränderter Kohlenhydrat-, Eiweiß- und Fettstoffwechsel schon vor der eigentlichen Manifestation der Kachexie vorhanden ist und sich progredient parallel zum Tumorwachstum verhält. Eine negative Stickstoffbilanz ist dabei ein verlässlicher Parameter für das Ausmaß des Nahrungsmangels. Eine Normalperson verliert beispielsweise täglich etwa 25 g Eiweiß, ein Krebspatient ohne Therapie etwa 50 $\mathrm{g}$ und unter Therapie sogar bis zu $80 \mathrm{~g}$ Eiweiß (14).

Tumorbedingte Nahrungsmangelzustände entstehen durch eine unter dem erforderlichen Tagesmindestbedarf liegende Nahrungszufuhr, durch Resorptionsstörungen des Dünndarms sowie durch einen erhöhten Katabolismus. Pathophysiologisch werden für die Entstehung der Tumorkachexie vor allem inflammatorische Zytokine wie Tumor-Nekrose-Faktor, Interleukin $1 \alpha$ und $ß$, Interleukin 6, Interferon $\gamma$ und ein Differenzierungsfaktor D verantwortlich gemacht (15), wobei der genaue Mechanismus und das Zusammenspiel der verschiedenen Faktoren unbekannt ist.
Appetitlosigkeit ist ein häufiges Symptom einer Tumorerkrankung, die durch die zytostatische Therapie noch verstärkt wird. Der Anorexie liegen sehr komplexe Prozesse zu Grunde (16). Fälschlicherweise sieht man häufig die Anorexie als den wesentlichen Grund für den Gewichtsverlust an. Dies konnte in Studien aber nicht nachgewiesen werden (17). Teilweise ist die Inappetenz offenbar durch eine Aminosäuren-Imbalance bedingt, deren Ausgleich aber nicht ohne Weiteres möglich ist, teils werden auch vom Tumor selbst Anorexine produziert (18). Wesentliche Momente für die Auslösung einer Anorexie bei Krebspatienten und den initialen Gewichtsverlust von 2,5 - 5kg sind psychischer Genese durch die Eröffnung der Diagnose, die Durchführung diagnostischer Maßnahmen und die Ungewissheit über den Ausgang der Erkrankung (19). Glukokortikoide sind zwar wirksame Appetitstimulanzien, aber Protein-katabol. Das Gestagen Megestrol ist ebenfalls appetitstimmulierend und Fett-anabol, erhöht aber nicht die Muskelmasse (20). Für Wachstumshormone existieren keine kontrollierten Studien bei Tumorpatienten. Studien mit Medikamenten und Substanzen, die eine antizytokine Wirkung entfalten, sind auf den Weg gebracht, um vor allem die Wirksamkeit von Thalidomid und der mehrfach ungesättigten $\omega$-3-Fettsäure in diesem Zusammenhang zu bestätigen (21).

\section{Enterale oder parenterale Ernährung?}

Es wird häufig argumentiert, dass die enterale Nahrungszufuhr, speziell bei Tumorpatienten physiologischer als eine parenterale Ernährung sei. Dieses Statement wurde jedoch nie klar definiert. Eine große Metaanalyse (22) bei nicht-tumortragenden Patienten kommt zu dem Schluss, dass eine enterale Ernährung weder aus physiologischer noch metabolischer Sicht oder hinsichtlich Funktion und Struktur des Gastrointestinaltraktes einen Vorteil gegenüber der parenteralen Ernährung bringt. Es

\footnotetext{
1 Herrn Professor em. Dr. Dr. h.c. mult. H.E. Bock zum 99. Geburtstag
} 
waren lediglich die Therapiekosten geringer und die Häufigkeit einer Sepsis bei Patienten mit akuten Abdominaltraumen reduziert. Ergebnisse mit einer „forcierten“ enteralen Ernährung bei Tumorpatienten sind entäuschend gewesen (23).

Untersuchungen bei Patienten mit chronischem Eiweißmangelzustand als auch bei experimentell induziertem Eiweißmangel bei sonst ausreichender Kalorienzufuhr (24) ergaben eine ausgedehnte Atrophie der resorbierenden Dünndarmschleimhaut und schwere funktionelle Resorptionsstörungen (25). Analoge anatomische und funktionelle Veränderungen wurden bei Patienten mit verschiedenen Neoplasien beschrieben (26). Diese bei Tumorpatienten bestehenden Resorptionstörungen, die sich mit zunehmendem Eiweißmangel verstärken, werden durch eine zytostatische, aber auch Strahlentherapie weiter erhöht (27). In einer randomisierten Sutdie bei knochenmarktransplantierten Patienten wurde gezeigt (28), dass eine Atrophie des Darmepithels durch die Zugabe von Glutamin zur prophylaktischen parenteralen Standard-Ernährung verhindert werden konnte. Diese Beobachtung wurde in einer Metaanalyse bestätigt (29). Eine enterale Ernährung ist zwar wegen der Erhaltung/Wiederherstellung der Integrität der Darmschleimhaut durchaus wünschenswert, jedoch unter bestimmten Bedingungen, wie stenosierenden Tumoren des Gastrointestinaltraktes bzw. bei vorliegender Übelkeit, Erbrechen, Geschmacksveränderung, Völlegefühl oder frühzeitigem Sättigungsgefühl der Patienten nicht immer praktikabel. Die Erfahrung aus der Praxis lehrt weiterhin, dass eine enterale Zusatzernährung aus Gründen der Compliance der Patienten häufig nur über kurze Zeiträume zu realisieren ist. In Übereinstimmung damit zeigt eine Metaanalyse aus dem Bereich der enteralen Zusatzernährung bei Morbus Crohn oft relativ hohe Abbruchraten, die u.a. durch das Auftreten von Diarrhoen oder Appetitlosigkeit zu erklären sind (30). Zudem ist die enterale Ernährung mit einer höheren Inzidenz einer inadäquaten Nährungsaufnahme assoziiert (31). So ist umgekehrt die parenterale Ernährung in dieser Situation als Ergänzung zu der häufig nicht ausreichenden normalen Ernährung bzw. einer enteralen Zusatzernährung geeignet, den Bedarf an Kalorien und Nährstoffen sicherzustellen.

Zumindest im fortgeschrittenen Stadium der Tumorerkrankung, scheint die parenterale Ernährung der enteralen überlegen, da ein positiver Effekt sowohl am Körpergewicht wie der Stickstoffbilanz sehr viel rascher eintritt (17).

kurzsgefasst: Vor- und Nachteile einer enteralen oder parenteralen Ernährung müssen sorgfältig gegeneinander abgewogen werden. Im fortgeschrittenen Stadium der Tumorerkrankung ist der parenteralen Ernährung der Vorzug zu geben.

\section{Wann sollte mit einer Ernährungstherapie begonnen werden?}

Tumorpatienten, die nicht kachektisch bzw. in höherem Maße unterernährt sind, können in der Regel zumindest eine Woche lang einen „Hungerzustand“ ohne Nebenwirkungen tolerieren. Deshalb sollte bei diesen Patienten in dieser Zeit keine parenterale Ernährung durchgeführt, sondern eine adäquate orale Nahrungsaufnahme angestrebt werden (32).
Ein bereits erlittener, schwerwiegender Verlust an Körpergewicht ist hingegen unter den veränderten Stoffwechselbedingungen eines onkologischen Patienten durch eine ernährungstherapeutische Maßnahme nur schwer auf den initialen $\mathrm{Zu}$ stand zurückzubringen. Jedoch kann mit dieser supportiven Maßnahme der Ernährungszustand von dem Zeitpunkt der Intervention an erhalten bzw. verbessert werden. Damit wird unseres Erachtens der potenziell von einer Mangelernährung bedrohte Patient möglichst frühzeitig diätetisch unterstützt. Unter Berücksichtigung der erhobenen Parameter zum Ernährungszustand sollte neben einer Diätberatung bzw. einer enteralen $\mathrm{Zu}-$ satzernährung auch die Möglichkeit einer zumindest zeitweisen frühzeitigen parenteralen Ernährung in Betracht gezogen werden. Ziel dieses Ansatzes ist also, die progrediente Entwicklung schwerer kachektischer Zustände von vornherein zu vermeiden. Der ärztlichen Kunst obliegt es hierbei, der häufig geäußerten Befürchtung des Patienten entgegenzuwirken, dass es sich bei der künstlichen Zusatzernährung um eine „letzte Maßnahme“ handele. Vielmehr ist sie geeignet, den Organismus zu stärken und damit einer Therapie der onkologischen Erkrankung zugänglicher zu machen.

kurzgefasst: Es ist wichtig, die psychische Hemmschwelle des Patienten gegen eine parenterale Ernährung abzubauen und letztere frühzeitig durchzuführen.

\section{Tumorwachstum und parenterale Ernähirung}

Schon frühzeitig wurde der Frage nachgegangen $(33,34)$, ob eine hochkalorische parenterale Ernährung das Ausmaß der Metastasierung bei Tumorpatienten fördert. Dabei wurde gefunden, dass eine verbesserte Ernährung des Patienten offenbar kein schnelleres Wachstum der Krebszelle zur Folge hat, sondern der Tumor nur in dem Maße proportional wächst wie auch die Eiweißmasse des Körpers zunimmt $(33,34)$. Möglicherweise erhöht die parenterale Ernährung während der Chemotherapie sogar die Sensitivität des Tumorgewebes gegenüber den Medikamenten. In einer randomisierten Studie (35) erhielten Patienten in schlechtem Ernährungszustand mit gastrointestinalen Tumoren präoperativ 10 Tage lang eine parenterale Ernährung mit oder ohne Chemotherapie. Bei den Patienten, die nur eine parenterale Ernährung erhielten, zeigte sich ein signifikant größerer Anstieg der Proliferation von Tumorzellen - gemessen an dem Anteil der Tumorzellen in S-Phase und dem DNA-Gehalt verglichen mit denen, die entweder zusammen mit der parenteralen Ernährung gleichzeitig eine Chemotherapie oder nur eine alleinige präoperative Chemotherapie erhielten. In der Kombination von parenteraler Ernährung plus gleichzeitiger Chemotherapie kompensierte die Chemotherapie die Stimulation der Tumorzellen durch die parenterale Ernährung. Bei der 10-tägigen präoperativen parenteralen Ernährung wurden $35 \mathrm{kcal}$ pro $\mathrm{kg}$ und Tag verabfolgt. Sie bestand zu 60\% aus hochprozentiger Glucose und 40\% Fettlösungen. Sollten auch bei anderen Tumorerkrankungen Tumore durch eine parenterale Ernährung zu erhöhtem Wachstum stimuliert werden, so wäre dies für eine gleichzeitige Chemo- oder Radiotherapie durchaus von Vorteil, da durch die Vergrößerung des proliferierenden Kompartiments die Wirksamkeit der Chemotherapie erhöht würde. 
In einer Metaanalyse von 19 Studien (35) konnte kein positiver Effekt der parenteralen Ernährung auf das Überleben von Patienten unter Chemo- oder Strahlentherapie gesehen werden. Dennoch kann diese Analyse nicht die Möglichkeit einer Verlängerung des Überlebens von Patienten unter/nach einer Knochenmarktransplantation (KMT) ausschließen $(32,35)$. Daher wird in der KMT-Situation von der American Gastroenterology Association keine Empfehlung für oder gegen eine parenterale Ernährung abgegeben und die Entscheidung dem behandelnden Arzt anheimgestellt.

\section{kurzgefasst: Das Tumorwachstum wird nicht durch die parenterale Ernährung in klinisch messbarer Weise stimuliert.}

\section{Immunität und parenterale Ernährung}

Ein Mangelernährungszustand bei Kindern wie Erwachsenen verändert die immunologischen Abwehrfunktionen und kann zur immunologischen Inkompetenz führen (38). Umgekehrt stellt eine konsequente Gewichtszunahme die zelluläre und humorale Abwehr voll wieder her (39). Die Überwachung zellulärer Immunreaktionen unter parenteraler Ernährung (14) liefert einen zuverlässigen Parameter für den jeweiligen Zustand des Körpereiweißstatus (40): Alle Patienten, bei denen eine parenterale Ernährung nicht ansprach, waren bei Testung anergisch und starben wenig später. Wie tierexperimentell gezeigt werden konnte (41), wurde durch Entzug von Aminosäuren, speziell Cystein, Valin, Tryptophan, Tyrosin und Phenylalanin aus der Nahrung sowohl die zelluläre Immunität gegen die Tumorzellen als auch die Produktion von Immunglobulinen reduziert.

\section{Vorteile einer parenteralen Ernährung bei Tumorpatienten}

Aus dem bisher Gesagten ergibt sich folgendes:

1. Tumorpatienten mit negativer Stickstoffbilanz haben - auch wenn keine Malignome im Bereich des Magen-Darm-Traktes vorliegen - im Allgemeinen schwere Resorptionsstörungen im Dünndarm, so dass eine Sondenernährung nur von begrenztem Nutzen ist.

2. Die meisten chemotherapeutischen Verfahren sind mit zahlreichen Nebenwirkungen wie Appetitlosikeit, Gewichtsverlust, Schwäche, Übelkeit, Erbrechen, Durchfällen und Zytopenien behaftet. Bei gleichzeitiger parenteraler Ernährung treten diese in weitaus geringerem Maße auf $(39,42-44)$.

3. Daher können höhere Dosen im Rahmen aggressiverer Schemata mit entsprechend besseren Erfolgsaussichten angewandt werden $(39,42,45,46)$.

4. Auch kachektische Patienten können eine adäquate Therapie erhalten, die ihnen sonst wegen des schlechten Allgemeinzustandes versagt bleiben müsste (47).

5. Eine mögliche Stimulierung von Tumorzellen mit einer Erhöhung in Teilung befindlicher Zellen könnte die Voraussetzungen für eine wirkungsvollere Chemotherapie verbessern (33-35).
6. Ernährungsbedingte Störungen der zellulären und humoralen Immunität werden behoben, so dass auch die dadurch verbesserte Infektabwehr eine aggressivere Behandlung auf einer internistischen Allgemeinstation ohne aufwendige Sterilbedingungen erlaubt $(14,39,41)$.

7. Die Verbesserung des Ernährungszustandes des Patienten unter Vermeidung lästiger, therapiebedingter Nebenwirkungen führt nicht zuletzt zu einer Besserung des Wohlbefindens und Hebung des Gemütszustandes, die wiederum die Kooperations-bereitschaft zu langwierigen therapeutischen Maßnahmen erleichtert $(48,49)$.

\section{Prinzip der parenteralen Ernährıung}

Im katabolen Zustand ist die prozentuale Abnahme der Körperzellmasse größer (36\%) als die Abnahme des Körpergewichtes (15\%) (50), d.h. der Verlust an Körpermasse bei alleiniger Messung des Körpergewichtes wird unterschätzt (20). Als klinisch bedeutsamster Parameter zur Beurteilung des Ernährungszustandes eines Patienten gilt immer noch das Verhältnis zwischen Körpergewicht und Körpergröße und die Veränderung dieses Verhältnisses im Verlauf der letzten Wochen und Monate. In allen klinischen Studien, speziell bei Tumorpatienten, wird nach wie vor $(35,51)$ eine Mangelernährung als ein Verlust von mehr als $10 \%$ des „optimalen“ Gewichtes definiert bei einem gleichzeitigen Serumalbuminspiegel unter $435 \mu \mathrm{mol} / \mathrm{l}$ (30 $\mathrm{g} / \mathrm{l})$. Diese Berechnungsmethoden für den Ernährungszustand sollten in Zukunft zu Gunsten des „Body Mass Index“ (BMI = Körpergewicht in kg dividiert durch das Quadrat der Körpergröße) verlassen werden (52). Über die genannten Beurteilungsparameter hinaus gilt auch die Bioimpedanzanalyse als valides Verfahren zur Beurteilung des Ernährungsstatus (53). Diese Methode erlaubt die nicht-invasive Bestimmung der unterschiedlichen Körperkompartimente. Sie ist jedoch mit höheren Investitionskosten verbunden und deshalb in Praxen nicht anzutreffen. Da Tumorerkrankungen häufig von einer Tumorkachexie begleitet sind (5), ist eine Verlaufsbeobachtung des Ernährungszustandes besonders wichtig.

Der früher verwendete Ausdruck ,intravenous hyperalimentation" war missverständlich, da die Therapie eine adäquate Ernährung und keine Überernährung zum Ziel hat. Er wurde durch den Begriff „total parenteral nutrition“ ersetzt. Die wesentlichen Energieträger einer parenteralen Ernährung sind Kohlenhydrate und Fette. Während die älteren Studien früher ausschließlich hochprozentige Kohlenhydrate als Energieträger verwendet und Fette nur bei länger dauernder parenteraler Ernährung zur Vermeidung eines Fettsäuremangels 1 mal pro Woche statt Kohlenhydrate verabreichten (54), besteht der Anteil an Kohlenhydraten als Energieträger heutzutage aus ca. 60-70\% und der an Fetten aus ca. 30-40\% $(35,55,56)$. In einer anderen Studie (57), allerdings an einer kleinen Fallzahl bei nicht-onkologischen Intensivpatienten, wurde sogar eine ausgeglichene Stickstoffbilanz bei einer Nicht-Protein-Energiezufuhr von 75\% Fett und $25 \%$ Glucose sichergestellt. Kohlenhydrate bzw. Fette sollten zusammen mit Aminosäuren (Eiweiß) in der Menge zugeführt werden, wie sie zur Vermeidung oder zum Ausgleich einer negativen Stickstoffbilanz und Aufrechterhaltung einer anabolen Stoffwechsellage erforderlich ist. Eine optimale Nahrungszu- 
fuhr, die sich an der Stickstoffbilanz orientiert, erfordert eine Kalorienzufuhr von 130-150\% des Basalstoffwechsels enteral ernährter und von 175\% parenteral ernährter Patienten (58). Ist der Kalorienbedarf gedeckt, so hängt eine Gewichtszunahme im Wesentlichen von der Eiweißzufuhr ab. Um eine volle Ausnutzung des zugeführten Eiweißes zu gewährleisten, ist es sinnvoll, die Energiequelle für diesen anabolen Prozess, den Zucker, bzw. die Fette gleichzeitig im Nebenschluss parenteral zuzuführen. Eine Gewichtszunahme tritt am zuverlässigsten dann ein, wenn die Eiweißzufuhr $1,5-2,0 \mathrm{~g} / \mathrm{kg}$ pro Tag beträgt (Stickstoff $\times 6,25=$ Eiweiß). Ein optimales Verhältnis von Stickstoff zu Kalorie beträgt $1 \mathrm{~g} \mathrm{~N}$ : 150-200kcal (628-837 kJ), also etwa $2000-2500 \mathrm{kcal} / \mathrm{d}$, entsprechend $8374-10467 \mathrm{~kJ}$ (5).

Am Beginn der Behandlung kachektischer Patienten steht die Rehydrierung, die behutsam über etwa eine Woche durchgeführt werden sollte. Das Blutvolumen, der onkotische Druck und die Serum-Eiweiß-Spiegel können dadurch normalisiert werden. Unabhängig davon tritt eine positive Stickstoffbilanz frühestens nach Ablauf einer Woche auf. In dieser Zeit sieht man klinisch durch die Verbesserung des onkotischen Druckes und Abnahme von Gewebsflüssigkeit einen weiteren geringen Gewichtsverlust, dann eine Gewichtsstabilisierung und schließlich eine wöchentliche Gewichtszunahme von etwa $2 \mathrm{~kg}$.

Insofern findet bei den drastisch rückläufigen Liegezeiten der Patienten (z.Zt. 6-7 Tage) im Krankenhaus die in früheren Jahren bei den meisten Chemotherapien routinemäßig durchgeführte totale parenterale Ernährung in der Klinik kaum noch Anwendung. Maßgeblich haben bei dieser Entwicklung auch die Versorgung der Tumorpatienten in Tageskliniken dazu beigetragen. Umgekehrt führt die Langzeit-Betreuung der Patienten zu Hause zu einer neuen Indikation der „home parenteral nutrition" (HPN) (59). Der Wert einer HPN ist seit langem bekannt (18), und es wurde in randomisierter Weise gezeigt (60), dass mit dieser Maßnahme bei Tumorpatienten im Endstadium ohne spezifische Chemo- oder Strahlentherapie eine signifikante Lebensverlängerung auftrat. Diese Beobachtung wurde kürzlich in einer doppel-blind, randomisierten Studie bestätigt (61).

kurzgefasst: Die parenterale Ernährung hat eine adäquate Ernährung und keine Überernährung zum Ziel. Früher wurden ausschließlich hochprozentige Kohlenhydrate als Energieträger verwendet, heute besteht der Anteil an Kohlenhydraten 60-70\% und der an Fetten zu 30-40\%.

\section{Glucose}

Physiologischerweise kommt es ohne Zufuhr von Kohlenhydraten zum Abbau von Körpereiweiß, aus dessen glucoplastischen Aminosäuren dann wieder Glucose gebildet wird. Dies ist ein sehr unökonomischer Vorgang, da aus $100 \mathrm{~g}$ Aminosäuren nur 57 g Zucker entstehen.

Der Bedarf glucoseabhängiger Gewebe an Zucker ist unter normalen Bedingungen sehr unterschiedlich, ebenso dessen Metabolisierung. Der Tagesbedarf des Zentralnervensystems liegt bei etwa 150 g Glucose, die vollständig zu Kohlendioxid und Wasser verbrannt wird. Erythrozyten, Granulozyten und das Knochenmark, Gewebe, welche durch die zytostatische Therapie mitgeschädigt werden, verbrauchen normalerweise etwa 2530 g Glucose, die anaerob bis zum Lactat abgebaut und dann im Cori-Zyklus durch Leber und Niere wieder zu Glucose resynthetisiert wird. Zudem benötigen Phagozyten für ihre Energiegewinnung ausschließlich Glucose als Substrat (62).

Bei Tumorpatienten liegt offenbar ein gestörter Glucose-Metabolismus vor: Es ist sowohl die Lactat-Produktion erhöht (63-65) als auch die Menge an Lactat, die wieder zu Glucose synthetisiert wird $(63,64,66)$. Diese erhöhte Aktivität des Cori-Zyklus, also die erhöhte Resynthese von Glucose aus Lactat in der Leber, wird als ein wesentlicher Mechanismus für den erhöhten Energieverbrauch bei Tumorpatienten angesehen $(67,68)$. Das Überwiegen der Lactatbildung verglichen mit der Oxidation von Glucose erhöht die Gluconeogenese (69). Der abnorm hohe Anteil am Recycling von Glucose wird nicht nur bei kachektischen $(65,70)$, sondern auch bei gut genährten Tumorpatienten gefunden (71). So gesehen wäre die exogene, parenterale Zufuhr von Glucose eher ein unökonomischer Vorgang (17), nichtsdestotrotz ist sie aber auch unter Verwendung hochprozentiger Glucose in praxi sehr effektiv, selbst bei weit fortgeschrittenen Malignomen hinsichtlich der Stickstoffbilanz und der Zunahme des Körpergewichtes (72).

Die Verwendung von Glucose im Infusionsplan hat gegenüber Fructose und in noch höherem Maße gegenüber Polyolen den Vorteil, dass sie von sämtlichen Geweben umgesetzt werden kann. Fructose sollte wegen der nicht seltenen Fructose-Intoleranz keine Verwendung mehr finden. Um metabolische Komplikationen zu vermeiden, hat es sich bei der Gabe hochprozentiger Glucose im Rahmen der totalen parenteralen Ernährung $(50 \%, 11)$ als zweckmäßig erwiesen, die Infusionsdauer auf mindestens 12, besser 24 Stunden festzulegen. Bei ausschließlicher Verwendung von hypertoner Glucose (Glucose System) als Ernergieträger verabfolgt man bei kachektischen Tumorpatienten im Rahmen der sog. Hyperalimentation, die vor allem in den USA, Frankreich und England angewendet wird, 0,5 g Glucose/ $\mathrm{kg}$ pro Stunde über den Infusomat. Dies ergibt bei einem $70 \mathrm{~kg}$ schweren Patienten 840 g Kohlenhydrat in 24 Stunden (73), was ungefähr dem vierfachen basalen Kohlenhydratbedarf entspricht. Da bei Stoffwechselgesunden bei Zufuhrraten von 1,5 g/ kg pro Stunde eine deutliche Glucosurie auftritt, sollten Zufuhrgrößen von $1 \mathrm{~g} / \mathrm{kg}$ pro Stunde nicht überschritten werden (74). Bei Diabetikern können auch schon niedrigere Dosen zu Hyperglykämien führen und somit die zusätzliche Gabe von Insulin notwendig machen. In Abhängigkeit von einer eventuell auftretenden Glykosurie sind Blutzuckerwerte bis 13,9 mmol/l $(2,5 \mathrm{ng} / \mathrm{dl})$ ohne Insulingaben tolerabel. In diesem Zusammenhang soll darauf hingewiesen werden, dass bei Tumorpatienten nicht selten eine Insulin-Resistenz beobachtet wird $(65,69,75)$, die schon frühzeitig im Verlauf der Erkrankung auftreten kann (76). Bei Einsatz von „halb“ Glucose und „halb“ Fettemulsion (Fettsäure System) sollte eine maximale Infusionsgeschwindigkeit von 0,25 g Glucose/kg pro Stunde eingehalten und maximal 5-6 g/kg und Tag bzw. ca. $400 \mathrm{~g}$ Glucose infundiert werden.

Gefahren einer parenteral verabfolgten, hochprozentigen $\mathrm{Zu}$ ckerlösung sind die Hyperglykämie mit Glucosurie und osmotischer Diurese bis hin zum hyperglykämischen, nicht-ketotischen Koma, Hypokaliämie durch die Einschleusung der Glucose in die Zelle und Abfall des anorganischen Serum-Phosphors 
(77). Letzteres führt zu einer Verminderung des intraerythrozytären Gehaltes an ATP und 2,3-Diphosphoglycerat, was eine Verschiebung der $\mathrm{O}_{2}$-Dissoziationskurve und somit eine erschwerte $\mathrm{O}_{2}$-Abgabe an die Gewebe zur Folge hat. Ein niedriges anorganisches Serum-Phosphor bedingt aber auch einen Abfall von ATP in den Granulozyten mit einer starken Abnahme chemotaktischer und phagozytärer Eigenschaften (78). Hierin scheint eine Erklärung für die schwere Beeinflussbarkeit von Sepsisfällen bei Patienten unter hochkalorischer, parenteraler Ernährung zu liegen.

\section{Fettsäuren}

Früher wurden im Rahmen der totalen parenteralen Ernährung bei Tumorpatienten als Energiequelle fast ausschließlich hochprozentige Kohlehydrate verwendet (54); Lipid-Infusionen waren nur angezeigt, wenn es darum ging, einen Mangel an essentiellen Fettsäuren und den damit verbundenen klinischen Symptomen bei länger dauernder parenteraler Ernährung zu vermeiden (79), oder wenn die Kalorienzufuhr durch Glucose allein nicht gedeckt werden konnte. Dieses Vorgehen wurde auch damit begründet, dass Fettsäuren per se die Stickstoffbilanz nicht verbessern könnten.

Später wurde dann bei postoperativen Patienten prospektiv und in randomisierter Weise gezeigt (80), dass 30\% der isokalorischen Kohlehydrat-Kalorien problemlos durch Fette ersetzt werden können und dabei keine signifikanten Unterschiede im Eiweißstoffwechsel auftraten: ob nun die totale parenterale Ernährung aus 25\% Glucose und 4,25\% Aminosäuren oder aus 15\% Glucose, Fettsäuren und 5\% Aminosäuren bestanden, die Stickstoffbilanz, das Gewicht und der Albumin-Spiegel im Serum besserten sich in beiden Gruppen gleichermaßen. Ein weiterer Vorteil dabei war, dass die zuvor erwähnten metabolischen Komplikationen bei ausschließlicher Verwendung von Glucose nicht mehr auftraten. Auch unter Verwendung einer länger dauernden totalen parenteralen Ernährung von polytraumatisierten und/oder septischen Patienten wurde beobachtet (81), dass sowohl bei ausschließlicher Verwendung von hypertonen Glucose-Infusionen (Glucose System) als auch bei Verwendung von „halb“ Glucose und „halb“ Fettemulsion (Fettsäure System) die Stickstoffbilanz positiv wurde und in beiden Gruppen nicht unterschiedlich war. Ein zusätzlicher Nutzen bei reduzierter Zufuhr von Glucose und gleichzeitig erhöhtem Fettanteil zur Deckung des Energiebedarfs besteht in einer Verkürzung der erforderlichen Infusionsdauer, da die Glucose quantitativ den größten Anteil aller Nährsubstrate ausmacht und dadurch die Mindestinfusionsdauer bestimmt wird. Dies ist vor allem für die heimparenterale Ernährung von Bedeutung. Die ideale Energiequelle für den Aminosäure-/Eiweißstoffwechsel im Rahmen der totalen parenteralen Ernährung (Glucose vs Fettsäure System) bei kritisch kranken Patienten bleibt jedoch kontrovers (82).

Die Dosierung der parenteral verabreichten Triglyceride bei Verwendung des Fettsäure-Systems sollte im Bereich von 1-1,5 $\mathrm{g} / \mathrm{kg}$ und Tag liegen. Als maximale Infusionsgeschwindigkeit ist eine Dosis von $0,15 \mathrm{~g} / \mathrm{kg}$ und Stunde wünschenswert, wobei zur Sicherstellung der besseren Verträglichkeit mit halber Rate eingeschlichen werden kann. Schließlich ist bei der Bemessung der Fettdosis auch der Triglycerid-Spiegel im Serum zu berücksichtigen, der $400 \mathrm{mg} / \mathrm{dl}$ nicht überschreiten sollte.
Bei Tumorpatienten sollte der veränderte Lipidstoffwechsel im Rahmen der parenteralen Ernährung berücksichtigt werden. Statt einer erhöhten Lipolyse scheint eher eine reduzierte Lipogenese bei Tumorpatienten typisch zu sein (83). Vor Einsetzen einer Tumorkachexie ist die Lipolyse nicht verändert $(69,84)$. Beim Vorliegen einer Unterernährung ist sie hingegen deutlich erhöht und übersteigt die Rate der Fettmobilisation. Es wurde berichtet (85), dass die exogene Zufuhr von Glucose die Fettsäure-Oxidation bei Tumorpatienten weniger als bei Gesunden unterdrückt. Während der parenteralen Ernährung unter Einschluss von Fettemulsionen ist bei gewichtsstabilen Tumorpatienten die Oxidation von Fettsäuren normal (84).

Wichtig bei der Wahl einer Fettemulsion ist ihr Gehalt an mittel- und langkettigen Fettsäuren: Zahlreiche Studien bei postoperativen Patienten (86-89), allerdings mit kleinen Fallzahlen, haben gezeigt, dass eine $20 \%$ Fettemulsion mit $50 \%$ mittelkettigen und $50 \%$ langkettigen Fettsäuren eine deutlich bessere Stickstoffbilanz zeigten als Fettlösungen mit ausschließlich langkettigen Fettsäuren. Ensprechend erfolgte eine Gewichtszunahme schneller. Daraus wurde geschlossen, dass mittelkettige Fettsäuren rasch hydrolisiert, zu Fettsäuren und Ketonkörpern oxidiert und dann verwertet werden können. Die Ketogenese und somit auch die Ketone im Plasma waren deutlich erhöht, die Respiration wurde nicht beeinflusst. Wegen der bei mittelkettigen Triglyceriden auftretenden Ketonämie und Ketonurie sowie Acetatämie sollten Lipidinfusionen bei latenten oder manifesten Acidosen gänzlich unterbleiben. Vorsicht ist ebenso bei einer Sepsis geboten, da Fette mit der Mikrozirkulation und der Aktivität der Mitochondrien interferieren und somit die Schockbereitschaft erhöhen können (90). $\omega$-3-Fettsäuren hingegen führen zu einer Verbesserung der Mikrozirkulation und Normalisierung des pulmonalvaskulären Widerstandes in der Schocklunge, zu einer Erhaltung der Splanchnikus-Durchblutung bei Sepsis und zu einer Abschwächung des Schadens nach Ischämie und Reperfusion (91).

Einige Autoren $(92,93)$ beobachteten bei Tumorpatienten eine deutliche Abschwächung der Tumorkachexie durch Anreicherung von $\omega$-3-Fettsäuren in der parenteralen Ernährung. Es wurde weiterhin beschrieben (94), dass der Gehalt an $\omega$-3-Fettsäuren im Fettgewebe bei lokal fortgeschrittenem Mammakarzinom ein unabhängiger Prognosefaktor ist und mit dem Ansprechen auf die Chemotherapie korreliert. Durch die Verbesserung der Mikrozirkulation in soliden Tumoren wird zudem die Radiosensitivität signifikant verbessert (95).

\section{Aminosäuren}

Ziel einer optimalen Infusionstherapie mit Aminosäuren ist vor allem der Ausgleich der negativen Stickstoffbilanz, also die Überführung aus einem katabolen in einen anabolen Zustand, und zusätzlich der Ausgleich eventuell vorhandener Imbalanzen im Aminosäuremuster.

Das physiologische Substrat für die parenterale Eiweißernährung sind ausschließlich adäquat zusammengesetzte Lösungen aus freien L-Aminosäuren. Sie müssen die acht klassischen essentiellen Aminosäuren, die drei „neuen“ (Arginin, Histidin und Prolin) sowie mindestens drei nicht-essentielle Aminosäuren unter Berücksichtigung von L-Glutaminsäure und L-Alanin ent- 
Tab.1 Rechenbeispiel für die Erstellung einer individuellen Rezeptur zur totalen parenteralen Ernährung (TPN).

Beispiel:

50 -jähriger Mann, $170 \mathrm{~cm}, 70 \mathrm{~kg}$, Flüssigkeitsbedarf $=35 \mathrm{ml} / \mathrm{kg} \times d=2450 \mathrm{ml} / \mathrm{d}$ Kalorienbedarf ca. $2500 \mathrm{kcal} / \mathrm{d}$

$\begin{array}{lll}\text { 1. Aminosäuren: } & \begin{array}{c}\text { Bedarf: } \\ \text { Berechnung: }\end{array} & \begin{array}{l}1,5 \mathrm{~g} / \mathrm{kg} \times \mathrm{d} \\ 1,5 \mathrm{~g} / \mathrm{kg} / \mathrm{d} \times 70 \mathrm{~kg}=105 \mathrm{~g} / \mathrm{d}=100 \mathrm{~g} / \mathrm{d} \\ (\mathrm{z} . \mathrm{B} . \text { Aminosäuren } 10 \% \mathrm{E} 1000 \mathrm{ml})\end{array} \\ & \begin{array}{l}\text { AS-kcal: } \\ 4,2 \mathrm{kcal} / \mathrm{g} \times 100 \mathrm{~g} / \mathrm{d}=420 \mathrm{kcal} / \mathrm{d}\end{array} \\ \begin{array}{l}\text { 2. Verteilung auf } \\ \text { Kohlenhydrate }\end{array} & \text { KH-kcal: Fett kcal }=60: 40\end{array}$

*Der zusätzliche Flüssigkeitsbedarf (vorzugsweise NaCl 0,9\% aus Kompatibilitätsgründen) verringert sich durch die Zusatzstoffe, durch die etwa $200 \mathrm{ml}$ Flüssigkeit zugeführt werden

halten. Glutamin, eine nicht-essentielle Aminosäure wird im katabol kranken Patienten essentiell. Sie verhindert eine Atrophie des Dünndarmepithels und verbessert die immunologische $\mathrm{Ab}$ wehrsituation durch einen positiven Effekt auf die Regeneration von neutrophilen Granulozyten (28). Klinisch wurde in einer Metaanalyse der Cochrane Collaboration gezeigt (29), dass Patienten nach KMT dann eine geringere Inzidenz positiver Blutkulturen, eine kürzere Krankenhausliegedauer und durch eine Verbesserung der digestiven und resorptiven Gegebenheiten seltener ein gastrointestinales Versagen zeigten, wenn Glutamin der parenteralen Ernährung zugesetzt wurde. Im Allgemeinen wird durch parenterale Zufuhr derartiger Aminosäurelösungen die tägliche Proteinzufuhr von etwa $1 \mathrm{~g} / \mathrm{kg}$ gewährleistet. Enthalten sie jedoch zu geringe Mengen an Methionin, so kommt es zu signifikant erniedrigten intra- und extrazellulären MethioninKonzentrationen und trotz allem ungünstiger Stickstoffbilanz (96).

Entsprechend dem erhöhten Eiweißumsatz von Tumoren und den dadurch bedingten hohen täglichen Eiweißverlusten (17) ist der Bedarf an Aminosäuren, die täglich gleichzeitig mit einem Energielieferant zugeführt werden müssen, hoch.

Metabolische Komplikationen durch parenteral verabfolgte Aminosäuren sind selten. Werden große Mengen von Aminosäuren-Hydrochloriden zugeführt, kann es zu einer hyperchlorämischen metabolischen Acidose kommen, die mit Kalium- oder Natriumacetat behoben werden kann. Enthalten die
Tab 2 Empfehlungen für die tägliche Elektrolytzufuhr.

\begin{tabular}{ll}
\hline Natrium & $1-2 \mathrm{mmol} / \mathrm{kg} \times \mathrm{d}$ \\
Kalium & $0,5-1,0 \mathrm{mmol} / \mathrm{kg} \times \mathrm{d}$ \\
Calcium & $0,1-0,3 \mathrm{mmol} / \mathrm{kg} \times \mathrm{d}$ \\
Magnesium & $0,1-0,2 \mathrm{mmol} / \mathrm{kg} \times \mathrm{d}$ \\
Phosphat & $0,2-0,5 \mathrm{mmol} / \mathrm{kg} \times \mathrm{d}$
\end{tabular}

Aminosäurelösungen unzureichende Mengen von Arginin oder verhältnismäßig zuviel Glycin, können besonders bei Patienten mit Leberstörungen, Hyperammonämien hervorgerufen werden (97).

\section{Verschiedene Zusätze}

Besonders bei Verwendung von elektrolytfreien Aminosäuren-, Kohlenhydraten und Lipidlösungen sollte an eine ausreichende Substitution mit Elektrolyten, Vitaminen und Spurenelementen gedacht werden. Vorausgesetzt, dass kein Mangelzustand vorliegt, benötigt der ausschließlich parenteral ernährte Patient pro kg Körpergewicht und Tag mindestens 1-2 mmol Natrium, 0,7-0,9 mmol Kalium, 0,1 mmol Calcium, 0,2 mmol Phosphor, $0,5 \mathrm{mg}$ Ascorbinsäure, $0,02 \mathrm{mg}$ Thiamin, $0,2 \mathrm{mg}$ Nicotinamid, $0,03 \mathrm{mg}$ Pyridoxin. Die Vitamine werden am besten in Form eines Multivitaminpräparates einmal täglich einer Glucose-Infusion beigefügt. Eisen $(0,3 \mu \mathrm{mol} / \mathrm{kg})$ und Folsäure $(3 \mu \mathrm{g} / \mathrm{kg})$ sollten einmal wöchentlich, Vitamin $B_{12}$ sollte alle 14 Tage substituiert werden.

Die Tab.1-4 geben Empfehlungen für die parenterale Ernährung.

\section{Vermeidung von Komplikationen bei der parenteralen Ernåhrung}

Zwei Hauptkomplikationen müssen bei der parenteralen Ernährung berücksichtigt werden: Katheterinfektion mit und ohne Sepsis und metabolische Komplikationen. Auf letztere wurde bei der Besprechung der einzelnen Substanzen bereits eingegangen. Ergänzend sollte dazu die Kreislaufbelastung durch ein Überangebot von Flüssigkeit bei herz- und niereninsuffizienten Patienten erwähnt werden. Der zuverlässigste Weg, metabolische Komplikationen aller Art zu vermeiden, ist die Aufrechterhaltung einer permanenten Zufuhr der Infusionslösungen über 12, besser 24 Stunden. Bei der heimparenteralen Ernährung ist aus Gründen der Praktibilität eine 12-stündige Infusionsdauer vorzuziehen. Die gefürchtetste Komplikation ist die Kathetersepsis. Die Kontamination von Lösungen steigt mit dem Quadrat der Zahl der Zusätze und (oder) der Liegedauer der Katheter. Einschränkend muss jedoch gesagt werden, dass dies großen Schwankungen unterliegt und ganz vom Können und der Disziplin des Krankenpersonals bei den notwendigen, absolut aseptisch durchzuführenden Manipulationen am zentralen Zugang abhängt. Damit gewinnt die Einhaltung unter hygienischen Gesichtspunkten geprüfter und standardisierter Vorgehensweisen in Form von sog. Pflegestandards eine zentrale Bedeutung bei der parenteralen Ernährung. Dies ist insbesondere im Rahmen der heimparenteralen Ernährung eine wichtige Voraussetzung. Zudem zeigen zwei große Untersuchungen $(48,98)$, dass selbst 
Tab.3 Vitaminpräparate für die parenterale Ernährung.

\begin{tabular}{|c|c|c|c|c|c|}
\hline Vitamin & $\begin{array}{l}\text { FrekaVit }^{\circledR} \text { wasserl. } \\
\text { Fresenius Kabi }\end{array}$ & $\begin{array}{l}\text { Soluvit }{ }^{\circledR} N \\
\text { Baxter }\end{array}$ & $\begin{array}{l}\text { Cernevit }^{\circledR} \\
\text { Baxter }\end{array}$ & $\begin{array}{l}\text { Multibionta }{ }^{\circledR} N \\
\text { Merck }\end{array}$ & $\begin{array}{l}\text { Zufuhr- } \\
\text { empfehlungen pro Tag * }\end{array}$ \\
\hline B1 (mg) & 3 & 2,5 & 3,51 & 10 & $3-4$ \\
\hline$B_{2}(m g)$ & 3,6 & 3,6 & 4,14 & 7,3 & $3-5$ \\
\hline$B_{6}(m g)$ & 4 & 4 & 4,53 & 12,35 & $4-6$ \\
\hline Nicotinamid (mg) & 40 & 40 & 46 & 40 & $40-50$ \\
\hline Pantothensäure (mg) & 15 & 15 & 16,15 & 25 & $10-20$ \\
\hline Ascorbinsäure (mg) & 100 & 100 & 125 & 100 & $100-300$ \\
\hline $\operatorname{Biotin}(\mu g)$ & 60 & 60 & 69 & --- & $60-120$ \\
\hline Folsäure $(\mu g)$ & 400 & 400 & 414 & - & $160-400$ \\
\hline \multirow[t]{2}{*}{$B_{12}(\mu g)$} & 5 & 5 & 6 & - & $1 \mathrm{mg}$ in 3 Monaten \\
\hline & $\begin{array}{l}\text { FrekaVit }{ }^{\circledR} \text { fettl. } \\
\text { Fresenius Kabi }\end{array}$ & $\begin{array}{l}\text { Vitalipid } \circledast \text { Adult } \\
\text { Baxter }\end{array}$ & & & \\
\hline $\begin{array}{l}\text { A } \\
\text { (Retinolpalmitat) }(\mathrm{mg})\end{array}$ & 1,941 & 1,941 & 2,06 & 1,65 & 1,8 \\
\hline$D(\mu g)$ & 5 & 5 & 5,5 & - & 5 \\
\hline $\begin{array}{l}E \\
(\propto \text {-Tocopherol-Äquivalent) (mg) }\end{array}$ & 10 & 9,1 & 10,2 & 5 & $20-40$ \\
\hline$K(m g)$ & 0,15 & 0,15 & - & - & $0,1-0,15$ \\
\hline
\end{tabular}

Tab.4 Empfehlungen für die tägliche Zufuhr von Spurenelementen bei TPN.

\begin{tabular}{|c|c|c|c|c|c|}
\hline $\begin{array}{l}\text { Spurenelemente } \\
\text { ( } \mu \mathrm{mol})\end{array}$ & $\begin{array}{l}\text { Tracitrans }^{\circledR} \text { plus } \\
\text { Fresenius Kabi }\end{array}$ & $\begin{array}{l}\text { Tracutil }^{\circledR} \\
\text { Braun }\end{array}$ & $\begin{array}{l}\text { Addel }^{\circledR} N \\
\text { Baxter }\end{array}$ & $\begin{array}{l}\text { Inzolen }{ }^{\circledR} H K \\
\text { Köhler Pharma }\end{array}$ & Zufuhrempfehlungen pro Tag * \\
\hline Zink & 100 & 50 & 100 & 48 & $21-75$ \\
\hline Kupfer & 20 & 12 & 20 & 28 & $7-23$ \\
\hline Eisen & 20 & 35 & 20 & - & $10-75$ \\
\hline Mangan & 5 & 10 & 5 & 8 & $3-14$ \\
\hline Molybdän & 0,2 & 0,1 & 0,2 & - & 0,2 \\
\hline Chrom & 0,2 & 0,2 & 0,2 & - & $0,2-0,3$ \\
\hline Selen & 0,4 & 0,3 & 0,4 & - & $0,25-0,8$ \\
\hline Jod & 1,0 & 1,0 & 1,0 & - & $0,8-1,2$ \\
\hline Fluorid & 50 & 30 & 50 & - & 49 \\
\hline
\end{tabular}

bei langdauernder totaler parenteraler Ernährung im Rahmen der häuslichen Betreuung von Tumorpatienten die Komplikationsrate durch Katheterinfektionen über einen Zeitraum von 12 Jahren signifikant gesenkt werden konnte und auch andere, etwa metabolische Komplikationen, die einen stationären Aufenthalt der Patienten nötig machten, gering waren. Die zuvor erwähnten Vorteile dieser unterstützenden Maßnahme überwiegen unserer Meinung nach bei weitem. Einschränkend soll jedoch erwähnt werden, dass die American Association of Gastroenterology (AGA) aufgrund ihrer Metaanalyse keine generelle Empfehlung für eine routinemäßige Anwendung einer parenteralen Ernährung unter Chemo- oder Radiotherapie abgibt, da die Komplikationsrate, speziell infektiöser Art in allen dort analysierten Studien zu hoch ist $(32,36)$. Sie hebt aber abschließend deutlich hervor (32), dass die analysierten randomisierten Studien nicht von hoher Qualität waren und in sämtlichen Studien, die in die Metaanalyse eingingen, kachektische Tumorpatienten ausgeschlossen waren (36). Daher würden die dort getroffe- nen Aussagen und Empfehlungen möglicherweise die Vorteile der parenteralen Ernährung unterschätzen.

\section{kurzgefasst: Die beiden Hauptkomplikationen bei der parenteralen Ernährung sind die Katheterinfektionen mit und ohne Sepsis sowie metabolische Komplikatio- nen. Bei der Verwendung des Glucosesystems ist dies die Glucosurie und Hyperglykämie, bei Verwendung des Fettsäuresystems die Ketonämie und Ketonurie sowie die Acetatämie.}

Autorenerklärung: Der Autor erklärt, dass er keine finanziellen Verbindungen zu einer Firma hat, deren Produkt in dem Artikel eine wichtige Rolle spielt (oder zu einer Firma, die ein Konkurrenzprodukt vertreibt).

Literatur im Internet unter www.thieme-connect.dle (98 Zitate, 1977-2002) 
Literatur

1 Jatoi A, Windschitl HE, Loprinzi CL. Dronabinol versus megestrol acetate versus combination therapy for cancer-associated anorexia: a North Central Cancer Treatment Group Study. J Clin Oncol 2002; 20: 567-573

2 Agteresch HJ, Rietveld T, Kerkhofs LGM. Beneficial effects of adenosine triphosphate on mutritional status in advanced bung Cancer patients: a randomized clincal trial. J Clin Oncol 2002; 20: 371-378

3 Davis MP, Dickerson D. Cachexia and anorexia: Cancer`s covert Killer. Support Care Cancer 2000; 8: 180-187

4 Body JJ. New hopes for the treatment of cancer anorexia and cachexia? Opinions Topics Support Care Oncol 2002; 42: 5-6

${ }^{5}$ Dewys WD, Begg C, Lavin PT, Band PR, Bennet JM, Bertino JR, Cohen MH, Douglas HO, Engstrom PF, Ezdinli EZ, Horton J, Johnson GJ, Moertel CG, Oken MM, Perlia C, Rosenbaum C, Silverstein MN, Skeel RT, Sponzo RW, Tormey DC and the Eastern Cooperative Oncology Group. Prognostic effect of weight loss prior to chemotherapy in cancer patients. Am J Med 1980; 69: 491-497

${ }^{6}$ Langstein HN, Norton JA. Mechanisms of cancer cachexia. Hematol Oncol Clin North Am 1991; 5: 103-123

7 Kern KA, Norton JA. Cancer cachexia. J Parent Ent Nutr 1988; 12: 286 298

8 Heber D, Byerly LO, Chlebowski RT. Medical abnormalities in the cancer patient. Cancer 1985; 55: 225-229

${ }^{9}$ Holroyde CP, Skutches CL, Boden G. Glucose metabolism in cachectic patients with colorectal cancer. Cancer Res 1984; 44: 5910-5913

${ }_{10}$ Lundholm K, Edstrom S, Karlberg I. Glucose turnover, gluconeogenesis from glycerol, and estimation of net glucose cycling in cancer patients. Cancer 1982; 50: 1142-1150

11 Norton JA, Maher M, Wesley R. Glucose intolerance in sarcoma patients. Cancer 1984; 54: 3022-3027

12 Jeevanandam M, Horowitz GD, Lowry SF. Cancer cachexia and protein metabolism. Lancet 1984; i: 1423-1426

${ }^{13}$ Vlassara H, Spiegel RJ, Doval DS. Reduced plasma lipoproteinlipase activity in patients with malignancy associated weight loss. Horm Metab Res 1992; 18: 698-703

${ }^{14}$ Solassol C, Joyeux H. Artificial gut in gastrointestinal cancer. Karger: Basel, In: Beiträge zur Infusionstherapie: Parenterale Ernährung, N Zöllner Ed. 1978: 178

15 McNamara MJ, Alexander HR, Norton JA. Cytokines and their role in the pathophysiology of cancer cachexia. J Parent Ent Nutr 1992; 16 : 50-55

16 von Meyenfeldt MF, Soeters PB. Anorexia in cancer. Karger: Basel, In: Nutrition in cancer and trauma sepsis. F Bozzetti, R Dionigi Eds. 1985 $54-67$

17 Holm E, Staedt U, Leweling H, Tokus M. Metabolism und artificial nutrition of patients with malignancy. SA J Clin Nutr 1993; 6: 10-21

18 Solassol C, Joyeux H. Ambulatory parenteral nutrition in hospital and at home. Little, Brown \& Co: Boston, In: Total parenteral nutrition; JE Fischer Ed. 1975

${ }^{19}$ Holland JCB, Rowland J, Plumb M. Psychological aspects of anorexia in cancer patients. Cancer Res 1977; 37: 2425-2428

${ }^{20}$ Keller U. Von der Katabolie zur Anabolie: Stoffwechselmediatoren und Therapieansätze. Akt Ernähr Med 2001; 26: 148-152

21 Gogos CA, Ginopoulos P, Salsa B. Dietary omega-3-polyunsaturated fatty acids plus vitamine $\mathrm{E}$ restore immunodeficiency and prolong survival for severely ill patients with generalized malignancy. Cancer 1998; 82: 395-402

22 Lipman TO. Is enteral nutrition really letter than parenteral nutrition? A look at the evidence. J Parent Ent Nutr 1998; 22: 169-182

${ }^{23}$ Body JJ. Metabolic sequaelae of cancers. Current Opin Clin Nutr and Metab Care 2002; 2: 339-344

24 Ramalingaswami V, Deo MG, Sood SK. Protein deficiency in Rhesus monkey. Nutritional Research Council. Washington D.C, Publication N. 843, National Academy of Sciences. 1961: 365

${ }^{25}$ Deo MG, Ramalingaswani V. Reaction of the small intestine to induced protein malnutrition in Rhesus monkeys. A study of cell population kinetics in the jejunum. Gastroenterol 1965; 49: 150-157

${ }^{26}$ Danon J, Gilat T. Absorption and enzyme studies in patients with malignancy. Digest 1968; 1: 316

27 Bounous G, Hugon JM, Gentile JS. Elemental diet in management of intestinal lesion produced by 5 -fluorouracil in man. Can J Surg 1971; 14: 298-304
${ }^{28}$ Sax HC. Clinical and metabolic efficacy of glutamine-supplemented parenteral nutrition after bone marrow transplantation, A randomized, double-blind, controlled study. J Parent Ent Nutr 1992; 16: 589590

${ }^{29}$ Murray SM, Pindoria S. Nutrition support for bone marrow transplant patients. (Cochrane Reriew). In: The Cochrane Liberary, Issue 3, 2002

30 Griffiths AM, Ohlsson A, Sherman PM, Sutherland LR. Meta-analysis of enteral nutrition as a primary treatment of active Crohn`s disease. Gastroenterol 1995; 108: 1056-1067

31 Woodcock NP, Zeigler D, Palmer MD, Buckley P, Mitchell CJ, MacFie J. Enteral versus parenteral nutrition: a pragmatic study. Nutrition 2001; 17: 1-12

32 Koretz RL, Lipman TO, Klein S. American Gastroenterological Association medical position statement: Parenteral nutrition. Gastroenterol 2001; 121: 966-969

33 Copeland EM, jr. MacFadyen, Dudrick SJ. Intravenous hyperalimentation in cancer patients. J Surg Res 1974; 16: 241-246

${ }^{34}$ Holm E. Ernährungstherapie bei Tumorkrankheiten: Wird der Tumor gefüttert? I Karger: Basel, n: Ernährung und Tumorerkrankungen. P. Schauder, ed. 1991: 454-476

35 Jin D, Philipps M, Byles JE. Effects of parenteral nutrition support and chemotherapy on the phasic composition of tumor cells in gastrointestinal cancer. J Parent Ent Nutr 1999; 23: 237-241

36 Koretz RL, Lipman TO, Klein S. American Gastroenterological Association: technical review on parenteral nutrion. Gastroenterol 2001; 121: $970-1001$

37 Weisdorf S, Lysne J, Wind D, Haake RJ, Sharp HL, Goldman A, Schissel K, Mc Glave PB, Ramsay NK, Kersey JH. Positive effect of prophylactic total parenteral nutrition on long-term outcome of bone marrow transplantation Transplant 1987; 43: : 833-838

38 Law DK, Dudrick KSJ, Abdou NJ. Immunocompetence of patients with protein-caloric malnutrition. Ann Int Med 1973; 79: 545-550

39 Copeland EM, MacFadyen BV, Rapp MA, Dudrick KSJ. Hyperalimentation and immune competence in cancer. Surg Forum 1975; 26: 138141

40 Blackburn GL, Maini BS, Bristrian BR, McDermott WV. The effect of cancer on nitrogen, electrolyte and mineral metabolim. Cancer Res 1977; 37: 2348-2353

${ }^{41}$ Jose DG, Good RA. Quantitative effects of nutritional essential amino acid deficiency upon immune response to tumor in nice. J Exp Med 1973; 137: 1-9

42 Copeland EM. Intravenous hyperalimentation and chemytherapy: An update. J Parent Ent Nutr 1982; 6: 236-239

43 Schwartz GF, G reen HL, Bendon ML, Graham WP, Blakemore WS. Combined parenteral hyperalimentation and chemotherapy in the treatment of disseminated solid tumors. Am J Surg 1971; 121: 169173

44 Copeland EM, Daly JM, Dudrick KSJ. Nutrition as an adjunct to cancer treatment in the adult. Cancer Res 1977; 37: 2451-2456

45 Souchon EA, Copeland EM, Watson P, Dudrick KSJ. Intravenous hyperalimentation as an adjunct to cancer chemotherapy with 5-fluorouracil. J Surg Res 1975; 18: 451-454

46 Lanzotti VJ, Copeland EM, George SJ, Dudrick SJ, Samuels ML. Cancer chemotherapeutic response and intravenous hyperalimentation. Cancer Chemother Rep 1975; 59: 437-439

47 Copeland EM, MacFadyen BV, Lanzotti VJ, Dudrick KSJ. Intravenous hyperalimentation as an adjunct to cancer chemotherapy. J Surg 1975; 129: 167-173

48 King LA, Carson LF, Konstantinidis N, House MS, Adcock LL, Prem KA, Twiggs LB, Cerra FB. Outcome assessment of home parenteral nutrition in patients with gynecologic malignancies: what have we learned in a decade of experience. Gyn Oncol 1993; 51: 377-382

49 Cozzaglio L, Balzola F, Cosentino F, DeCicco M, Fellagra P, Gaggiotti G, Gallitelli L, Giacosa A, Orban A, Fadda M, Gavazzi C, Pirovano F, Bozzetti F. Outcome of cancer patients receiving home parenteral mutrition. J Parent Ent Nutr 1997; 21: 339-342

50 Wilmore DW. Catabolic illness: Strategies for enhancing recovery. N Engl J Med 1988; 12: 286-298

51 Blackburn GL, Thornton PA. Nutritional assessment of hospitalized patients. Med Clin Noth Am 1979; 63: 1103-1115

52 Behrendt W. Klinisch relevante Parameter zur Beurteilung des Ernährungszustandes. Akt Ernähr Med 1999; 24: 14-19

${ }^{53}$ Fischer H, Lembcke B. Die Anwendung der bioelektrischen Impedanzanalyse (BIA) zur Beurteilung der Körperzusammensetzung und des Ernährungszustandes. Inn Med 1991; 18: 13-17 
54 Jehn U, Wohlrab A, Wilmanns W. Hinweise zur parenteralen Ernährung von Tumor-Patienten. Deutsch Med Wochenschr 1979; 15: 536-540

55 Weisdorf SA, Lysne J, Wind D, Haake RJ, Sharp HL, Goldman A, Schissel K, Mc Glave PB, Ramsay NK, Kersey JH. Positive effect of prophylactic total parenteral nutrition on long-term outcome of bone marrow transplantation. Transplant 1987; 43: 833-838

${ }^{56}$ Sikora SS, Ribeiro U, III. Kane JM, Landreneau RJ, Lembersky B, Posner MC. Role of nutrition support during induction chemoradiation therapy in esophageal cancer. J Parent Ent Nutr 1998; 22: 18-21

57 Baker JP, Detsky AS, Stewart S, Whitwell J, Marliss EB, Jeejeebho KN. Randomized trial of total parenteral nutrition in critically ill patients: metrabolic effects of varying glucose-lipid ratios as energy source. Gastroenterol 1987; 87: 53-59

${ }^{58}$ Hallowell E, Sasvary D, Bistrian BR, Soroff HS, Blackburn GL. Factors determining optimal nutritional support in defined formula diet therapy (Abstract). Clin Res 1975; 23: 591

${ }^{59}$ Howard L. Home parenteral nutrition in patients with a cancer diagnosis. J Parent Ent Nutr 1992; 16: 93-99

60 Solassol C, Joyeaux H. Artificial gut with complete nutritive mixtures as a major adjuvant therapy in cancer patients. Acta Chir Scand 1979; : 186-188(Suppl 494

${ }^{61}$ Charuhas PM, Fosberg KL, Bruemmer B, Aker SN, Leisenring W, Seidel $\mathrm{K}$, Sullivan KM. A double-blind randomized trial comparing outpatient parenteral nutrition with intra-venous hydration: effect of resumption of oral intake after marrow transpantation. J Parent Ent Nutr 1997; 21: 157-161

62 Cahill GF, Felig E, Marliss B. Some physiological principles of parenteral nutrition: Grune \& Stratton, New York-London, In: Body fluid replacement in surgical patient, LG Fox and GG Nahas eds. 1970: 286

63 Holroyde CP, Axelrod RS, Skutches CL, Haff AC, Paul P, Reichard GA. Lactate metabolism in patients with metastatic colorectal cancer. Cancer Res 1979; 39: 4900-4904

${ }^{64}$ Holroyde CP, Reichard GA. Carbohydrate metabolism in cancer cachexia. Cancer Treat Rep 1981; 65: 55-59(Suppl 5

65 Shaw JH, Humberstone DA, Holdaway C. Weight loss in patients with head and neck cancer: Malnutration or tumor effect? Aust NZJ Surg 1988; 58: 505-509

${ }^{66}$ Waterhouse C. Lactat metabolism in patients with cancer. Cancer 1974; 33: 66-71

${ }^{67}$ Gold J. Proposed treatment of cancer by inhibition of gluconeogenesis. Oncol 1968; 22: 185-207

68 Young VR. Energy metabolism and requirements in the cancer patient. Cancer Res 1977; 37: 2336-2347

69 Douglas RG, Shaw JHF. Metabolic effects of cancer. Br J Surg 1990; 77 : 246-254

70 Eden E, Edström S, Bennegard K, Schersten T, Lundholm K. Glucose flux in relation to energy expenditure in malnourished patients with and without cancer during periods of fasting and feeding. Cancer Res 1984; 44: 1718-1724

${ }^{71}$ Long CL, Merrick H, Grecos G, Blakemore WS, Geiger J. Glucose metabolism and colorectal carcinoma. Metabolism 1990; 39: 494-501

72 Kohlschütter B, Jehn U. Die parenterale Ernährung bei aggressiver Chemotherapie verschiedener Neoplasien. Onkol 1978; 1: 31-34

73 Schumer W. High caloric solutions in traumatized patients. Grune \& Stutton: New York-London, In: Body fluid replacement in the surgical patient. CL Fox and GG Namas, eds. 1970: 326

74 Heuckenkamp PU. Kohlehydrate-Teilaspekte der Energieversogung. Karger: Basel, In: Beiträge zur Infusionstherapie. Parenterale Ernährung. N Zöllner ed. 1978: 3

${ }^{75}$ Lundholm K, Holm G, Schersten T. Insulin resistance in patients with cancer. Cancer Res 1978; 38: 4665-4670

${ }^{76}$ Norton JA, Maher M, Wesley R, White D, Brennan MF. Glucose intolerance in sarcoma patients. Cancer 1984; 54: 3022-3027

77 Dudrick SJ, MacFadyen BV, van Buren CT, Ruberg RL, Maynard AT. Parenteral hyperalimentation, metabolic problems and solutions. Ann Surg 1972; 176: 250

78 Craddock PR, Yawata Y, von Sauten L, Gilberstadt S, Silvis S, Jacob HS. Acquired phagocyte dysfunction: A complication of the hypophosphatemic of parenteral nutrition. New Engl J Med 1974; 290: 1403

79 Dudrick SJ, MacFadyen BV, Souchon EA, Englert DM, Copeland EM. Parenteral nutrition techniques in cancer patients. Cancer Res 1977; 37: 2440
80 Paluzzi M, Meguid MM. A prospective randomized study of the optimal source of nonprotein calories in total parenteral nutrition. Surg 1987; $102: 711-717$

81 Nordenstrom J, Askanazi J, Elwyn DH, Martin P, Carpentier YA, Robin AP, Kinney JM. Nitrogen balance during total parenteral nutrition: glucose vs fat. Ann Surg 1983; 197: 27-33

82 de Chalein TM, Michell WL, O'Kneefe SJ, Ogden JM. The effect of fuel source on amino acid metabolism in critically ill patients. J Surg Res 1992; 52: 167-176

83 Jeevanandem M, Horawitz GD, Lowry SF, Brennan MF. Cancer cachexia and the rate of whole body lipolysis in man. Metabolism 1986; 35 : 304-310

${ }^{84}$ Shaw JHF, Wolfe RR. Fatty acid and glycerol kinetics in septic patients and in patients with gastrointestinal cancer. Ann Surg 1987; 205: 368-376

85 Waterhouse C, Kemperman JH. Carbohydrate metabolism in subjects with cancer. Cancer Res 1971; 31: 1273-1278

${ }^{86}$ Lunstedt B, Deltz E, Kahler M, Bruhn A. Randomized study comparing long-chain (LCT) and medium-chain (MCT) triglycerides as caloric carriers in postoperative nutritional therapy. Infusionsther Klin Ernaehr 1987; 14: 61-64

87 Jiang ZM, Zhang SY, Wang XR, Yang NF, Zku Y, Wilmore D. A comparison of medium-chain and long-chain triglyceride in surgical patients. Ann Surg 1993; 217: 175-184

88 Ball MJ. Parenteral nutrition in the critically ill: use of a medium chain triglyceride emulsion. Intensive Care Med 1993; 19: 89-95

89 Lai H, Chen W. Effects of medium-chain and long-chain triacylglycerols in petiatric surgical patients. Nutrition 2000; 16: 401-406

90 Liljedahl St. Parenterale Ernährung und andere therapeutische Maßnahmen bei schweren Verbrennungen. Karger: Basel, In: Beiträge zur Infusionstherapie. Parenterale Ernährung. N Zöllner ed. 1978: 48

91 Pscheidl E. Fettemulsionen - Strukturierte Lipide und Omega-3-Fettsäuren. Akt Ernähr Med 2001; 26: 62-69

92 Chira T, Nishio K, Oke Y. Improvement by eicosanoids in cancer cachexia induced by LLC-116 transplantation. J Cancer Res Clin Oncol 1996; 122: 711-715

93 Wigmore StJ, Ross JA, Falconer JSt, Plester CE, Tisdale MJ, Carter DC, Fearon $\mathrm{KCH}$. The effect of polyunsaturated fatty acids on the progress of cachexia in patients with pancreatic cancer. Nutrition 1996; 12: 527-530

94 Bougnoux P, Germain E, Chajes V, Hubert B, Lhuillery C, Le Floch O, Body G, Calais G. Cytotoxic drugs efficacy correlates with adipose tissue docosahexaenoic acid level in locally advanced breast carcinoma. Brit J Cancer 1999; 79: 1765-1769

95 Baronzio G, Freitas J, Griffini P, Bertone V, Pacini F, Mascaro G, Razzini E, Gramaglia A. Omega-3-fatty acids can improve radioresponse modifying tumor interstitial pressure, blood rheology and membrane peroxidability. Anticancer Res 1994; 14: 1145-1154

96 Jürgens P. Aminosäuren in der parenteralen Ernährung. Springer: Berlin-Heidelberg-New York, In: Fettemulsionen in der parenteralen Ernährung. A Wretlind, R Frey, K Eyrich, H Makowski eds. 1977: 41

97 Shenkin A, Wretlind A. Allgemeine Aspekte hinsichtlich der intravenösen Ernährung von Krebspatienten. Infusionsther 1978; 5: 156165

98 Johnston DA, Pennington CR. Home parenteral intrition in Tayside 1980-1992. Scottish Med J 1993; 38: 110-111 